UCRL-JC- 125760

PREPRINT

\title{
Fragmentation of Biomolecules Using Slow Highly Charged Ions
}

\author{
C. Ruehlicke, D. Schneider, R. DuBois, R. Balhorn
}

This paper was prepared for submittal to the

14th International Conference on the Application of Accelerators in Research and Industry Denton, TX

November 6-9, 1996

\section{November 1996}

- This is a preprint of a paper intended for publication in a journal or proceedings. Since changes may be made before publication, this preprint is made available with the understanding that it will not be cited or reproduced without the

- permission of the author. 


\section{DISCLAMMER}

This document was prepared as an account of work sponsored by an agency of the United States Government. Neither the United States Govermment nor the University of California nor any of their employees, make any warranty, express or implied, or assume any liability or responsibility for the accuracy, completeness, or usefulness of any information, apparatus, product, or process disclosed, or represents that its specific commercial products, process, or service by trade name, trademark manufacturer, or otherwise, does not necessarily constitute or imply its endorsement recommendation, or favoring by the United States Government or the University of California. The views and opinions of authors expressed herein do not necessarily state or reflect those of the United States Government or the University of California, and shall not be used for advertising or product endorsement purposes. 


\title{
FRAGMENTATION OF BIOMOLECULES USING SLOW HIGHLY CHARGED IONS
}

\author{
Christiane Ruehlicke ${ }^{1}$, Dieter Schneider ${ }^{1}$, Robert DuBois ${ }^{2}$, Rodney Balhorn ${ }^{3}$ \\ 'Physics and Space Technology, ${ }^{3}$ Biology and Biotechnology, Lawrence Livermore Natl. Lab., Livermore, \\ CA 94550, 2 Department of Physics, University of Missouri-Rolla, Rolla, MO 65401
}

\begin{abstract}
We present first results of biomolecular fragmentation studies with slow highly charged ions (HCI). A thin layer of the tripeptide RVA was deposited on gold targets and irradiated with slow (few $100 \mathrm{keV}$ ) ions, e.g. $\mathrm{Xe}^{50+}$ and $\mathrm{Xe}^{15+}$, extracted from the LLNL EBIT (electron beam ion trap). The secondary ions released upon ion impact were mass analyzed via Time-Of-Flight Secondary-Ion-Mass-Spectrometry (TOF-SIMS): The results show a strong dependence of the positive and negative ion yields on the charge state of the incident ion. We also found that incident ions with high charge states cause the ejection of fragments with a wide mass range as well as the intact molecule ( $345 \mathrm{amu}$ ). The underlying mechanisms are not yet understood but electron depletion of the target due to the high incident charge is likely to cause a variety of fragmentation processes.
\end{abstract}

\section{INTRODUCTION}

Interactions between slow $(\sim 2 \mathrm{keV} / \mathrm{amu})$ highly charged ions $(\mathrm{HCl})$ with biomolecules are being investigated. Currently the emphasis of the studies is on exploring the basic mechanisms of the interaction, e.g. breakup, in particular in polypeptides. These experiments might reveal a new method of degrading biomolecules. A potential application could be the fragmentation of proteins, which is used in peptide mapping and sequencing. The availability of proteases, which are commonly used for protein fragmentation, is very limited, therefore there is a demand for additional fragmentation techniques.

Studying the impact of HCI upon biomolecules also is an extension of studies of $\mathrm{HCI}$ solid surface interaction, which have shown that $\mathrm{HCI}$ impact causes the emission of a large number of electrons from the surface $(1,2)$, enabling a variety of secondary processes to take place.

The ions used in these studies are produced by an electron beam ion trap (EBIT) $(3,4)$ and charge states up to $50+$ have been used. The high potential energy (few 100 $\mathrm{keV}$ ) enhances the effect of electronic interactions with solids or molecules as compared to the nuclear interactions that occur in the collisions. The potential energies of the ions are comparable to their kinetic energy, giving rise to new effects.

\section{EXPERIMENTAL}

The target, the polypeptide RVA, which consists of a sequence of the aminoacids Arginine, Valine and Alanine, was synthesized using a PS3 Peptide Synthesizer (Rainin
Instruments) and purified by reversed-phase high performance liquid chromatography. Aliquots of the peptide $(50 \mu \mathrm{l}$ of a $10 \mathrm{mg} / \mathrm{ml}$ solution in water) were deposited on flat gold disks and allowed to dry for $15 \mathrm{~h}$ in the presence of a desiccant. The targets were clamped onto a sample ladder and mounted in the experimental area within one day. TOF-SIMS was performed in a high vacuum $\left(10^{-10}\right.$ Torr) chamber equipped for surface analysis at the end of the ion extraction beamline on EBIT. Xe gas was injected via a ballistic jet into the trap region of EBIT and $\mathrm{Xe}$ ions with charge states ranging from $15+$ to $50+$ were extracted at kinetic energies of $700 \mathrm{keV}$ or $105 \mathrm{keV}$ resp.

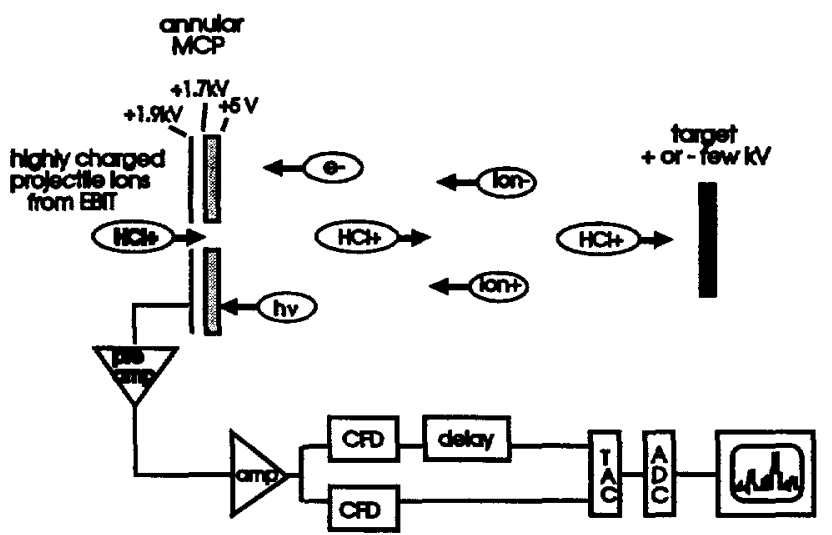

FIGURE 1. The Time-Of-Flight system used in our experiments is shown in this figure. Secondary ions and electrons ejected from the target are accelerated towards the microchannelplate detector. The target is biased positive or negative depending on which spectra are taken. Start and stop pulses are determined due to their pulse heights and arrival time at the detector. 
The fluxes were ca. 1000 ions $/ \mathrm{s}$, where the $\mathrm{Xe}^{15+}$ was extracted in pulses of a few ms length at $2 \mathrm{~Hz}$ and the $\mathrm{Xe}^{50+}$ was extracted in a continuous mode. The TOF spectra were obtained using a TOF-SIMS spectrometer (Fig. 1) (5). Secondary ions are accelerated between the target and a channelplate detector at voltages of a few $\mathrm{kV}$. For negative ions the start signal was taken from electrons emitted from the sample upon individual ion impact, while protons and in rare cases $\mathrm{H}_{2}^{+}$provided the start signal in the positive case. The stop signals were given by the secondary ions. The flight time of the secondary ions increases with charge and decreases with the mass at the same time.

\section{RESULTS AND DISCUSSION}

Examples of TOF-SIMS spectra are shown in Fig. 2. For both the negative and the positive secondary ion spectra the yields are much higher for $\mathrm{Xe}^{50+}$ being the primary ion compared to $\mathrm{Xe}^{15+}$. This general trend has also been observed with incident ions of other charge states. The $\mathrm{Xe}^{50+}$ spectra show a wide mass range of secondary ion peaks, many of which are below the background intensity in - the corresponding $\mathrm{Xe}^{15+}$ spectra. This demonstrates a higher sputtering efficiency for ions with high charge states. The large numbers of very low mass ions, e.g. $\mathrm{H}, \mathrm{C}$, and $\mathrm{O}$ compounds, mostly stem from contaminant on the surface rather than from the peptides and are common in these spectra regardless of the target material. While these compounds as well as atomic ions of alkali metals and halides used during sample preparation dominate the spectra up to masses of ca. 100, most larger mass fragments are unique to the peptide sample and appear to be molecular fragments. Both the positive and the negative spectra show a contribution at the mass of the intact molecule in the $\mathrm{Xe}^{50+}$ spectra, which indicates that it is also possible to lift intact molecules with both negative and positive excess charge from a solid surface. The occurrence of even higher masses suggests that the intact molecule combines with other fragments, possibly $\mathrm{Na}$. Since we can not distinguish between different charge states with the current setup, we assume all fragments to be singly charged. In addition to these peaks and a wide distribution of fragments in both the positive and negative spectra the negative spectrum also shows some distinctive peaks at masses 113,155 and 249 amu. The $\mathrm{Xe}^{15+}$ spectra both show fragments up to ca. $100 \mathrm{amu}$, higher mass peaks are not removed efficiently enough to be distinguished from the background counts on the spectra. This is true also for the peak corresponding to the intact molecule, whose mass position is marked in the positive spectrum.

The underlying mechanisms for breakup and ablation are not yet understood but it is assumed that different processes occur, which originate in the large electric field induced by the high ion charge. This high field could remove binding electrons, therefore creating fragments which leave the molecule.

However it is also possible that the $\mathrm{HCI}$ interacts
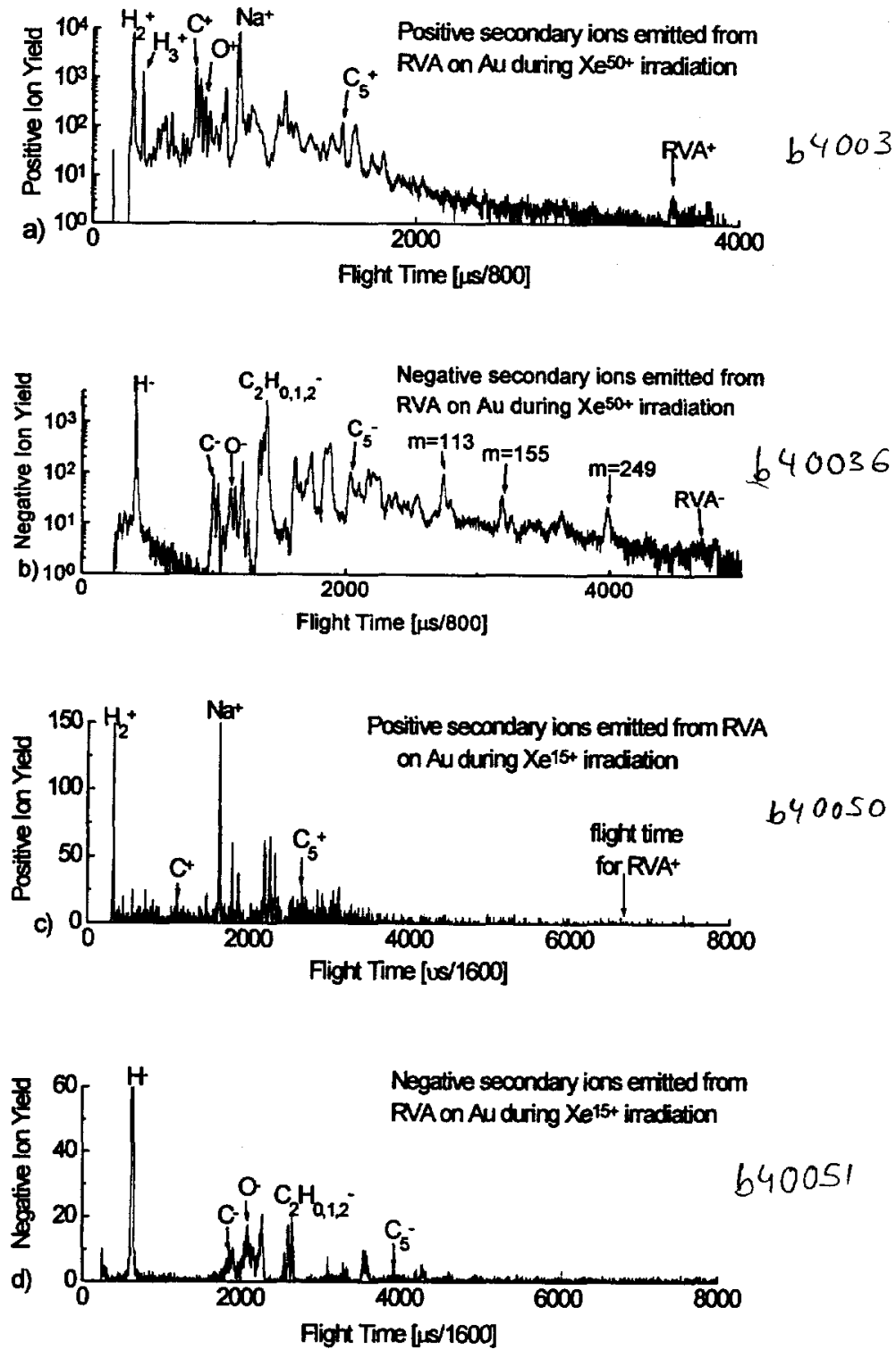

FIGURE 2. TOF-SIMS spectra showing the negative and positive secondary ion yields for projectile ions $\mathrm{Xe}^{50+}$ and $\mathrm{Xe}^{1 \mathrm{st}}$ impact on the tripeptide RVA deposited on a Au substrate. The yields, which were normalized to $10^{6}$ incident projectile ions, are plotted as a function of flight times of the secondary ions. A linear background was subtracted from the spectra. A few characteristic peaks are marked in all spectra, in c) we indicate the position where the $\mathrm{RVA}^{+}$peak would be expected, while d) actually shows a shorter mass range than the other spectra.

primarily with the solid substrate, upon which the molecules are deposited, which then interacts with the sample: e.g. in solid substrate sputtering the ejection of large clusters is ascribed to a shock wave in the solid generated by the ion (6). This might cause the desorption of intact molecules.

While the structure, binding and adhesion of the polypeptide molecules differs significantly from the solid surfaces, some of the responses to $\mathrm{HCI}$ impact are similar, e.g. the higher secondary ion yields and occurrence of high mass clusters due to $\mathrm{HCI}$ impact have been observed in sputtering solid surfaces with $\mathrm{HCI}$ as well (5). 


\section{CONCLUSIONS}

First results of fragmentation studies of biological molecules by $\mathrm{HCI}$ are presented. The TOF-SIMS spectra of the tripeptide RVA show the ablation of the intact molecule from a gold surface due to $\mathrm{HCI}$ impact as well as the fragmentation into a series of smaller fragments. The sputtering efficiency was shown to increase with increasing projectile ion charge and negative and positive secondary ion spectra were found to show different fragment yields. Some parallels can be drawn to interactions that occur between solid surfaces and $\mathrm{HCI}$ but the underlying mechanisms are not yet understood.

\section{ACKNOWLEDGMENT}

This work has been performed under the auspices of the U.S. Department of Energy by Lawrence Livermore National Laboratory under Contract No. W-7405-ENG-48.

\section{REFERENCES}

1. McDonald, J. W., Schneider, D., Clark, M. W. and Dewitt, D. Phys. Rev. Lett. 68, 2297 (1992)

2. Kurz, H., Aumayr, F., Winter, H. P., Schneider, D., Briere and M. A., McDonald, J. W., Phys. Rev., A49, 4693 (1994)

3. Levine, M. A., Marrs, R. E., Henderson, J. R., Knapp, D. A., and Schneider, M. B., Physica Scripta T22, 17 (1988)

4. Schneider, D. H., et al., Phys. Rev. A44, 3119 ( 1991)

5. Schneider, D. H. G. and Briere, M. A., Physica Scripta 53, 228 (1996)

6. Parilis, E. S. et al., "Atomic Collisions on Solid Surfaces" (North Holland, Amsterdam 1993), 353 


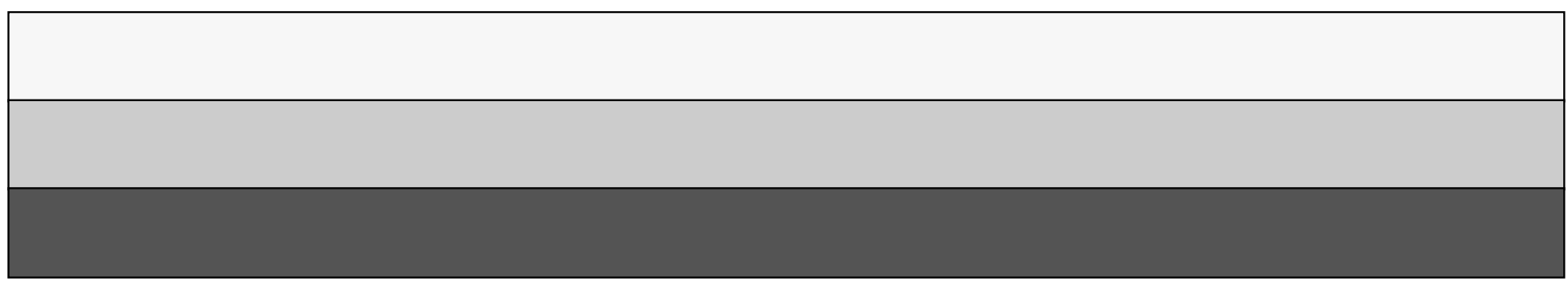

